\title{
La alteridad levinasiana y su analogía con el escepticismo ${ }^{1}$ \\ Levinasian alterity and its analogy with skepticism
}

Recibido: 20 de agosto de 2016 - Revisado: 24 de noviembre de 2016 - Aceptado: 18 de mayo de 2017.

Juan Carlos Aguirre García ${ }^{2}$

\section{Resumen}

Este artículo se propone evaluar la idea del filósofo Emmanuel Levinas según la cual propone que la alteridad es semejante al escepticismo, así como determinar si, en efecto, la alteridad levinasiana está comprometida con el escepticismo o si por el contrario, es una posibilidad para vislumbrar una racionalidad renovada en la discusión sobre lo humano. Para ello se expone la analogía entre alteridad y escepticismo; posteriormente, se revisan algunas respuestas dadas por estudiosos del autor; finalmente, se concluye indicando algunos modos de leer la alteridad levinasiana a través de la lente de una racionalidad renovada en el contexto de las Ciencias humanas.

\section{Palabras clave}

Racionalidad, alteridad, Levinas, “otrismo”, escepticismo.

\begin{abstract}
This article proposes to evaluate the idea of the philosopher Emmanuel Levinas according to which he proposes that alterity is similar to skepticism, as well as to determine if, in fact, Levinasian alterity is compromised with skepticism or if, on the contrary, it is a possibility to glimpse a renewed rationality in the discussion about the human. For this the analogy between alterity and skepticism is exposed; later, some answers given by scholars of the author are reviewed; finally, it is concluded by indicating some ways of reading the Levinasian alterity through the lens of a renewed rationality in the context of human Sciences.
\end{abstract}

\section{Keywords}

Rationality, alterity, Levinas, "otrismo”, skepticism.

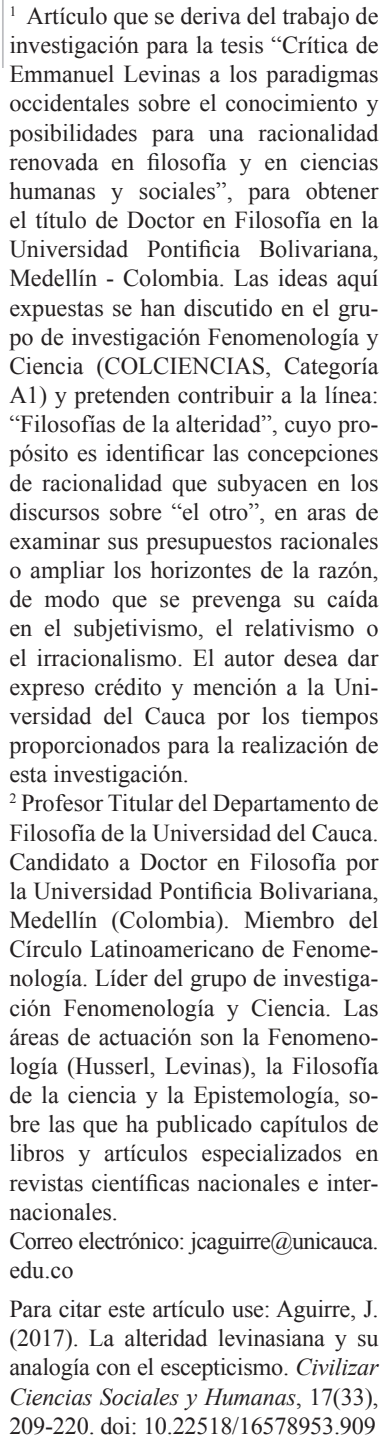

${ }^{1}$ Artículo que se deriva del trabajo de investigación para la tesis "Crítica de Emmanuel Levinas a los paradigmas occidentales sobre el conocimiento y posibilidades para una racionalidad renovada en filosofía y en ciencias humanas y sociales", para obtener el título de Doctor en Filosofía en la Universidad Pontificia Bolivariana, Medellín - Colombia. Las ideas aqu expuestas se han discutido en el grupo de investigación Fenomenología y Ciencia (COLCIENCIAS, Categoría A1) y pretenden contribuir a la línea: "Filosofías de la alteridad", cuyo propósito es identificar las concepciones de racionalidad que subyacen en los discursos sobre "el otro", en aras de examinar sus presupuestos racionales o ampliar los horizontes de la razón, de modo que se prevenga su caída en el subjetivismo, el relativismo o el irracionalismo. El autor desea dar expreso crédito y mención a la Universidad del Cauca por los tiempos proporcionados para la realización de esta investigación.

${ }^{2}$ Profesor Titular del Departamento de Filosofia de la Universidad del Cauca. Candidato a Doctor en Filosofía por la Universidad Pontificia Bolivariana, Medellín (Colombia). Miembro del Círculo Latinoamericano de Fenomenología. Líder del grupo de investigación Fenomenología y Ciencia. Las áreas de actuación son la Fenomenología (Husserl, Levinas), la Filosofía de la ciencia y la Epistemología, sobre las que ha publicado capítulos de libros y artículos especializados en revistas científicas nacionales e internacionales.

Correo electrónico: jcaguirre@unicauca. edu.co

Para citar este artículo use: Aguirre, J. (2017). La alteridad levinasiana y su analogía con el escepticismo. Civilizar Ciencias Sociales y Humanas, 17(33), 209-220. doi: 10.22518/16578953.909 


\section{Introducción}

La obra de Emmanuel Levinas está enmarcada en su obsesión por fundamentar la ética como filosofía primera; aunque tal vez esta no fuera su intención inicial ${ }^{1}$. De acuerdo con Critchley (2004), "La gran idea de Levinas es que la relación con el otro no se puede reducir a la comprensión, y que esta relación es ética; esto estructura la experiencia de lo que pensamos que es un yo o un sujeto" (p. 25). No sorprende entonces, que tal obra haya sido acogida en marcos estrictamente éticos, o a lo sumo políticos ${ }^{2}$; sin embargo, su propuesta ha quedado confinada a discusiones muy puntuales que, en la mayoría de casos, eluden asuntos latentes, necesitados de clarificación, no solo para considerar la solidez de los argumentos, sino también para evaluar la viabilidad de sus ideas. ¿Acaso antes de señalar que la "relación [con el otro] es ética", no afirma Critchley que dicha relación "no se puede reducir a la comprensión"? Ahora bien, ¿puede entenderse la irreductibilidad del otro a la comprensión en los modos usuales de inteligibilidad?; ¿acaso la propuesta levinasiana requerirá una revisión de los presupuestos tradicionales y una exploración o ampliación de las maneras clásicas de pensar los asuntos, especialmente los atinentes a lo humano?; ¿exigirán las formulaciones de Levinas una racionalidad renovada? La aparente osadía de algunas de sus afirmaciones así parece requerirlo.

Un ejemplo de esos asuntos latentes lo constituye la posición denominada por Aikin y Simmons (2009) como "otrismo" (Otherism), susceptible de autorrefutación. Estos autores reconstruyen del siguiente modo la posición levinasiana:

Emmanuel Levinas sostiene que la relación ética, en cuanto está más allá del ser, está también más allá de la tematización (es decir, no se puede articular en el lenguaje de acuerdo con conceptos generales que estén situados fundamentalmente en el ser). Llamaremos a esta posición "otrismo" (Otherism). Al describir la relación -lo cual es necesario si va a defender que el "otrismo" está en primer lugar-, debe tematizar lo no tematizable; en otros términos, tiene que dar una explicación de aquello que resiste toda explicación. Aparentemente, esta idea se autorrefuta; esto es, si es verdadera, implica su propia falsedad, pues lo que no se puede referenciar debe elegirse y exhibirse como imposible de referenciar ( $\mathrm{p}$. 29).

Esta acusación surgió muy pronto y su expresión magistral se condensa en el célebre texto "Violence et Métaphysique", de Derrida (1964/1967), con el cual este filósofo y amigo de Levinas cuestiona sus ideas (publicadas hasta la fecha), y especialmente, la lectura que hace de Husserl y Heidegger. Línea a línea, Derrida le muestra a Levinas que en su intento por destruir la "conceptualidad tradicional" (que es, en últimas, el tejido que constituye el "discurso filosófico"), queda preso de ella:

El extranjero de El Sofista, que parece romper con el eleatismo, como Levinas, en nombre de la alteridad, sabe que la alteridad no se piensa más que como negatividad, no se dice, sobre todo, más que como negatividad [...] y que, a diferencia del ser, lo otro es siempre relativo, se dice pros eteron, lo que no le impide ser un eidos [...] Por su parte, Levinas se rehusaría a asimilar "el otro" al eteron del que se trata aquí. ¿Pero cómo pensar o decir «el otro» sin la referencia $[. .$.$] a la alteridad del eteron en$ general? (pp. 186-187).

En el fondo, lo que Derrida indica a Levinas es que además del riesgo de autorrefutación, su "menosprecio del discurso" (p. 170) es una "especie de gráfica inaudita, dentro de la cual la conceptualidad filosófica no tendría más que una función" (p. 163); en últimas, esto significaría que sin la conceptualidad filosófica, Levinas no estaría ya en el terreno de la filosofía.

Algunos estudiosos de Levinas consideran que la obra principal que nos ocupa en este artículo es la escrita en 1974, Autrement qu'être 
ou au-delà de l'essence (en adelante se mencionará esta obra como $A E$ ), es su respuesta a las críticas expuestas por Derrida. Ahora bien, en vez de actualizar la polémica punto por punto, en este artículo se propone explorar la analogía con el escepticismo (sección que cierra esta obra); si bien es un posible modo de responder a Derrida, la analogía podría significar mucho más que eso: la viabilidad misma de la propuesta filosófica de Levinas y, ante todo, la posibilidad de una racionalidad renovada. Lo anterior se desarrollará por secciones, así: en primer lugar se explicará en qué consiste la analogía; en segundo lugar se reseñarán algunas interpretaciones relevantes que se le han dado y, por último, se expondrán las conclusiones, en las que se descubren algunas consecuencias atinentes a la posibilidad de una racionalidad renovada, especialmente en el contexto de las Ciencias humanas.

\section{El escepticismo y la analogía levinasiana: Entre la alteridad y el escepticismo}

A lo largo de la historia de la Filosofía, el escepticismo es uno de los movimientos más interesantes; podría decirse que ha sido, en varias ocasiones, el detonante de la reflexión filosófica. Al respecto dice Klein (2015):

Gran parte de la epistemología ha surgido o bien en defensa o bien en oposición a las distintas formas de escepticismo. Ciertamente, las diferentes teorías del conocimiento se pueden clasificar de acuerdo con sus respuestas al escepticismo; por ejemplo, los racionalistas podrían tomarse como escépticos acerca de la posibilidad del conocimiento empírico, pero no son escépticos con relación al conocimiento a priori; por su parte, los empiristas podrían tomarse como escépticos acerca de la posibilidad de un conocimiento a priori, pero no con relación al conocimiento empírico. Además, concepciones sobre muchos problemas filosóficos tradicionales, por ejemplo: el problema de las otras mentes o el problema de la inducción, pueden verse como formas restringidas de escepticismo que plantean que no podemos tener conocimiento de ninguna proposición en ningún dominio particular donde creemos que tenemos conocimiento (Traducción propia, párr. i).

En vista de la riqueza de las versiones y las múltiples formas que adopta el escepticismo, sería pretencioso tratar de condensarlas en unas cuantas líneas; no obstante, para tener en mente un marco general de discusión, puede sostenerse que "en filosofía, los argumentos escépticos, en general, se proponen mostrar que no conocemos o no estamos justificados para creer muchas cosas que asumimos ordinariamente que conocemos o que creemos razonablemente" (Lemos, 2007, p. 131).

A juzgar por sus escritos ${ }^{3}$, el conocimiento de Levinas sobre el tema del escepticismo no superaba la información básica que debe tener todo filósofo. Aparte de las consideraciones en su período de juventud y escasas menciones en obras filosóficas y judías, Levinas solo usa recurrentemente el término en $A E$.

En su juventud, especialmente en su primera obra, Théorie de l'intuition dans la phénoménologie de Husserl (1930/2001), Levinas (2001) retoma lo esencial de la respuesta husserliana contra el naturalismo. Luego de exponer las tesis husserlianas, concluye que "El escepticismo lleva ese nombre precisamente por no conceder el valor de ser a lo que conocemos y por guiarse por una idea de ser que expresa la existencia de la cosa bajo un solo aspecto: a partir de su pretendida independencia con respecto a los fenómenos que la constituyen" (p. 49).

De acuerdo con esto, y que es la línea argumentativa que Levinas mantiene en este tratado, el escepticismo está basado en errores, siendo el principal de ellos la pretensión de escindir los fenómenos y la conciencia constituyente. En tal sentido, desenmascarando los errores que el escéptico sostiene -lo cual puede hacerse, según Levinas, acogiendo la perspectiva fenomenológica husserliana-, no habría razones para adoptarlo o sucumbir a sus encantos. 
No obstante, al final del libro, luego de afirmar el error del escepticismo, su carácter de absurdo, e incluso "la contradicción que caracteriza a todo escepticismo" (Levinas, 2001, p. 197), el filósofo proclama algo sorprendente (valga advertir, no desplegado argumentativamente): "Sin embargo, esta refutación formal del escepticismo no es suficiente y Husserl parece reconocerlo" (p. 197). En efecto, en las páginas finales, aunque reafirma la pertinencia de Husserl para la refutación de un escepticismo que se respalde en el naturalismo, Levinas encuentra que el intento husserliano se queda corto frente a la "situación histórica del hombre", sobre la cual afirma "Esta estructura de conciencia [...] no ha sido estudiada por nuestro autor, al menos en las obras publicadas hasta el momento. En ellas no encontramos el problema de la relación entre la historicidad de la conciencia y su intencionalidad, su socialidad y su personalidad" (p. 221) . En consecuencia, queda abierta una puerta que explorará ampliamente solo en la sección "Escepticismo y razón" de $A E^{5}$.

Es justamente en esta sección donde podría establecerse la relación entre la alteridad levinasiana y la analogía con el escepticismo. Levinas comienza con un párrafo que sintetiza la concepción que tiene de razón (occidental): "La razón, en la cual los términos diferentes están presentes, es decir, son contemporáneos en el sistema, también es el hecho que están presentes a la conciencia, en tanto la conciencia es representación, comienzo, libertad" (Levinas, 1974, p. 210). Si se rastrea la obra de Levinas, puede encontrarse que hay consistencia en la reconstrucción que hace de la epistemología occidental, lo mismo que en la crítica que elabora para intentar superarla. En este apartado específico, el filósofo (Levinas, 1974) enuncia, a modo de conjetura, el terreno donde podría horadarse esta tradición:

El problema consiste solamente en preguntarse si el comienzo está al comienzo, si el comienzo como acto de conciencia no está ya precedido por lo que no se puede sincronizar, esto es, por lo que no podrá estar presente, por lo no representable, si no hay una anarquía más antigua que el comienzo y la libertad (p. 211).

Es obvio que, de acuerdo con su tesis de la alteridad, su respuesta será afirmativa. Para Levinas, "el otro" rompe esa prioridad (carácter de primero, de origen, de comienzo) de la conciencia, así que estaría antes de los principios que la conciencia establece. Además sostiene que "El otro como otro, como prójimo, nunca es igual en su presencia a su proximidad" (Levinas, 1974, p. 211); es decir, "el otro", en su proximidad no es un fenómeno o un tema para la conciencia (en todo caso no sería un fenómeno o tema en el sentido habitual en el que la conciencia tematiza los fenómenos), y así lo expresa: "La proximidad es una diferencia, una no coincidencia, una arritmia en el tiempo, una diacronía refractaria a la tematización, refractaria a la reminiscencia que sincroniza las fases de un pasado" (p. 211). En consecuencia, "el otro" es "un opuesto, un rebelde" a la tematización, a aceptar "idea, opinión o costumbre" 6 que brotan de esa tematización. En últimas, esto significa que las palabras utilizadas para abordar "al otro", y los discursos que se tejen en torno a él siempre son insuficientes, como indica aquí: "¡El inenarrable! -el otro que pierde su rostro de prójimo en la narración-. Relación indescriptible, en el sentido literal de este término; inconvertible en historia, irreductible a la simultaneidad de un escrito que registra o presenta resultados" (p. 211). Esta idea es relevante para confrontar la tradición epistemológica occidental y para postular una "inteligibilidad de la proximidad" (l'intelligibilité comme proximité) cuya significación excede los propósitos de este artículo.

Es en este contexto que Levinas (1974) plantea la analogía entre la alteridad y el escepticismo al afirmar que "Si la razón pre-original de la diferencia y de la no-indiferencia, de la responsabilidad -bello riesgo- conserva su significación, la pareja escepticismo y refutación del escepticismo debe hacer su aparición" 
(p. 213). Levinas identifica dos características presentes en el escepticismo: su "retorno periódico" y su "refutación"; esto es, la filosofía no puede deshacerse completamente y para siempre de los reclamos escépticos; pese a las sofisticadas refutaciones a las que ha sido sometido por parte de la filosofía, el escepticismo siempre se renueva, y aunque su recurrente presencia, que perturba el flujo tranquilo de la historia de la filosofía y su sincronía, ella siempre emerje como vencedora, como lo afirma Levinas (1974): "La historia de la filosofía occidental no ha sido más que la refutación del escepticismo tanto como la refutación de la trascendencia" (p. 214), pero, al fin y al cabo, y en un sentido eminente, a la filosofía le pertenece la última palabra: “¿No pertenece a la filosofía la última palabra?" (p. 213).

La relación que ve Levinas (1974) entre el escepticismo y su filosofía de la alteridad radica, fundamentalmente, en la diacronía (diachronie), y es su respuesta a la racionalidad occidental para la renovación del discurso filosófico: "la diacronía como el tiempo de la filosofía" y como "la fuerza invencible del escepticismo" (p. 214). Ambos serían refractarios ante la pretensión de un discurso filosófico encerrado en la sincronía; ambos espetarían a la razón que las cosas no son tan simples como parecen (o, mejor, como aparecen).

Ahora bien, ¿sería posible un discurso filosófico sostenido por la diacronía?; ¿acaso la definición misma de discurso, en cada una de sus acepciones, no alude ya a tematización y, por tanto, inexorablemente, a sincronía? Cuando Levinas (1974) afirma que "El lenguaje es ya escepticismo" (p. 216), ¿no es un claudicar de la razón? Él es consciente del riesgo que esto implica:

Si el discurso filosófico se rompe, se retira de la palabra y murmura, se habla, habla sin embargo de eso, y del discurso en el cual hasta entonces hablaba, al cual retorna para expresar su retraimiento provisional. ¿No estamos nosotros mismos en este momento a punto de borrar la salida que busca nuestro ensayo y encerrando en un círculo nuestra posición por todas partes? Las palabras excepcionales mediante las cuales se dice la huella del paso y la extravagancia del acercamiento -Uno, Dios- se convierten en términos, vuelven al vocabulario y se ponen a disposición de los filósofos, en lugar de desmontar el lenguaje filosófico (p. 215. Traducción ligeramente modificada).

La analogía entre alteridad y escepticismo no pretende reducir el predicamento; por el contrario, lo radicaliza. No obstante, pueden verse los puntos en discusión: por un lado, la insistencia en que el lenguaje excede los límites del pensamiento, "dejando sobreentender sin hacer entender nunca; se trataría de una implicación, de un sentido distinto a aquel que surge en el signo de la simultaneidad del sistema o de la definición lógica de un concepto" (Levinas, 1974, p. 215); por otro lado, el reconocimiento de que el discurso filosófico pervive, en parte por la asociación filosofía-Estado, y pese al reto escéptico que le reprueba que las estructuras que lo sustentan no son "el esqueleto último del sentido" (Levinas, 1974, p. 217); más aún, en su persistencia, se vuelve el trasfondo del que se alimentan tanto el escepticismo como la alteridad, pues en últimas, sin el estallido de la unidad de la apercepción trascendental, dice Levinas (1974), "tampoco se podría otro modo que ser" (p. 218).

\section{Algunas interpretaciones de la analogía}

La recepción de este apartado conclusivo de $A E$ ha despertado mucho interés entre los estudiosos de Levinas. Algunas tendencias interpretativas han evaluado el uso que Levinas da al término escepticismo; otras han estudiado la integración del apartado en el conjunto de la obra levinasiana; unas más aportan conclusiones que arrojan propuestas novedosas que amplían los horizontes reflexivos. Esta sección considerará brevemente tales tendencias. 
En primer lugar, y en concordancia con lo dicho anteriormente, pareciera que Levinas no era un experto en el tema del escepticismo; en tal sentido, se pretende exponer algunas precisiones al respecto. Lamarche (2008) acusa a Levinas de reducir el escepticismo a "dogmatismo negativo", esto es, "la tesis que "todos los enunciados filosóficos son falsos" o que "nada se puede conocer" (p. 166). De acuerdo con Lamarche, a este tipo de 'escepticismo' podría acusársele de autorrefutación, como lo hace Levinas, habiendo otros tipos de escepticismo que no sucumben a tal problema. En efecto, Lamarche se propone mostrar cómo el escepticismo pirrónico sale avante de la crítica de autorrefutación, entre otras razones, por no plantear ninguna tesis y porque este tipo de escepticismo "no es esencialmente una empresa epistemológica sino más bien una agogé, un estilo de vida" (p. 167) ${ }^{7}$. En similar sentido se encuentra el trabajo de Aikin y Simmons (2009), quienes apelan a la figura de Sexto Empírico (uno de los más importantes representantes del escepticismo), aunque con conclusiones mucho más prometedoras para la consideración epistemológica de Levinas. Según estos autores, "la analogía entre otrismo y escepticismo es desastrosa por dos razones: fuerza a la filosofía levinasiana a sostener una posición que no puede y no debería sostener; $\mathrm{y}$ hace del escepticismo algo que simplemente no es" (p. 45). Si bien esta primera tendencia interpretativa obligaría a tachar de infortunada la analogía establecida por Levinas, no es en sí misma incontrovertible; por ejemplo, la acusación de "dogmatismo negativo" podría, si se consideran otros elementos aparte de la autorrefutación, no ajustarse adecuadamente a lo planteado por Levinas; del mismo modo, quizás una consideración más abierta del asunto podría establecer semejanzas, más que diferencias, entre Levinas y el tipo de escéptico que defiende Lamarche. Así mismo, contentarse con endilgar a Levinas un desconocimiento de las discusiones detalladas del escepticismo e invalidar con ello su analogía, podría situar la discusión en terrenos poco propicios para el análisis fértil, cerrando prematura e injustamente la cuestión.
Precisamente por lo anterior, puede explorarse una segunda tendencia interpretativa que intenta, sin pasar por alto la posible falta de conocimiento preciso de Levinas con respecto al escepticismo, escudriñar el apartado confrontándolo con el resto de la obra del autor. Valga advertir que esta ha sido la tendencia expuesta por la mayoría de teóricos. Un caso emblemático lo constituye Bernasconi (1991), quien parte de dos supuestos indiscutibles en el texto de Levinas: a) el escepticismo y su refutación son tomados por Levinas como metáfora o modelo; y, b) no hay razones para concluir que Levinas esté adoptando una posición escéptica, ni siquiera que haga alguna evaluación al respecto (p. 152). Tal y como el contexto del libro lo sugiere, Bernasconi ubica la discusión en la distinción hecha por Levinas entre "el decir" (le dire) y "lo dicho" (le dit), y más específicamente, en la sincronía que rompe y la diacronía que inaugura: "La diacronía del decir, donde el decir escapa a la presencia, otorga las bases para el intento levinasiano por explicar cómo el escepticismo se escapa de la refutación que concede en otro nivel" (Bernasconi,1991, p. 150,-el énfasis es del autor del presente artículo-). No obstante, aunque la analogía se sostiene en la diferencia "el decir - lo dicho", Levinas no confunde "el decir" con el escepticismo; a lo sumo, la analogía funciona en tanto el escepticismo se encuentra divorciado de la tendencia de la filosofía occidental "con relación a un decir que se agota en las cosas dichas" (p. 151).

De acuerdo con esto, Levinas establecería una analogía entre su propuesta de la alteridad y el escepticismo, en tanto este, si bien ha sido reo de refutaciones a lo largo de la historia del pensamiento (primer nivel en el que se toma el escepticismo como parte del juego entre la razón desafiada y la razón capaz de superar el desafío), ha retornado una y otra vez, denunciando que no está en el mismo nivel en el que se le pretende refutado, a la vez que le reprocha a la filosofía su fracaso. Ahora bien, si es cierto que la primera línea argumentativa de Levinas, 
según Bernasconi, tiene como meta discutir las condiciones bajo las cuales la filosofía significa su verdad, condiciones puestas en cuestión por el escéptico, también es cierto que esto no significa una destrucción de la verdad. Según Bernasconi (1991), "el escepticismo, en la explicación de Levinas, permanece en el amor a la verdad. El escepticismo y la refutación del escepticismo son una "pareja"; La filosofía no puede separarse del escepticismo" (p. 151). En consecuencia, de acuerdo con la primera intención levinasiana reconstruida por Bernasconi, tanto el escepticismo como la filosofía de la alteridad irrumpen en el discurso filosófico desafiándolo, más que destruyéndolo; lo perturban una y otra vez, mas necesitan de él, así sea para demostrarle a la verdad filosófica que no puede encontrar paz en sus fundamentos, pues dichos fundamentos trascienden lo que ella puede darse libremente.

Además de esta interpretación de Bernasconi, los trabajos de Hutchens (2004) y Morgan (2007) son relevantes en esta segunda tendencia. El primer teórico defiende la tesis que el apartado en cuestión es un ejercicio para decir algo escépticamente: 'Levinas está 'diciendo' escépticamente lo que es el escepticismo y no solo tematizando lo que es el escepticismo" (Hutchens, 2004, p. 56. Énfasis en el texto original). Con base en lo anterior, el decir escéptico rompería la alternancia o sincronía entre la verdad filosófica y su puesta en cuestión por el escepticismo; puesta en cuestión que la verdad filosófica vuelve tema para su refutación, continuando en un círculo perenne. El decir algo escépticamente es analizado por Hutchens en la distinción que establece Levinas entre anfibología y apofansis, la cual se establece fundamentalmente en el acto dialógico:

El lenguaje en el diálogo tiene dos aspectos. Por un lado, el diálogo tiene la pretensión, mediante un propósito inteligible, de clarificar el sentido por medio de la tematización racional. Por otro lado, el lenguaje desborda los sentidos presentados mediante la tematización racional y, en consecuencia, resiste el esfuerzo dialógico por establecer un propósito inteligible (Hutchens, 2004, p. 56).

Al primer aspecto del lenguaje en el diálogo, Hutchens (2004), siguiendo a Levinas, lo denomina "reducción de la traición" y al segundo aspecto lo llama: "traición de la reducción"; al igual que el segundo nivel del que hablaba Bernasconi, esta traición de la reducción sería función del escepticismo y, a su vez, no totalmente refutable. Ahora bien, el que Hutchens sitúe la discusión del apartado en el contexto del diálogo, permite recuperar uno de los temas levinasianos que podrían ser útiles al momento de contrarrestar cierto escepticismo local -por ejemplo el relacionado con las otras mentes $-^{8}$, a saber, la expresión.

Para los propósitos de este artículo, es importante considerar el párrafo conclusivo del texto de Hutchens (2004): "En un nivel, es obvio que [Levinas] no pretende que su orientación sea epistemológica; pero en otro nivel, tiene que ser epistemológica para describir el esquema reducción de la traición / traición de la reducción" (p. 66). En otras palabras, por más que trate de eludir la cuestión, la propuesta levinasiana tendrá que lidiar con asuntos epistemológicos ${ }^{9}$.

El trabajo de Morgan (2007), por su parte, es un fino ejercicio de análisis, con la ventaja de culminar relacionando el apartado "Escepticismo y razón" con ciertas discusiones acerca de los límites del pensamiento y del lenguaje en la tradición analítica contemporánea. Del trabajo de este autor, se destacan tres ideas fundamentales: en primer lugar, lo que se ha nombrado aquí como "otrismo" es visto por Morgan como un problema metodológico: "Levinas reconoce este reto [de Derrida] como un reto serio; fue una de las razones para escribir De otro modo que ser. Él llama a este un 'problema metodológico"' (p. 304) ${ }^{10}$.

En segundo lugar, Morgan (2007) demuestra que no hay una simple analogía entre 
el escepticismo y la alteridad levinasiana: "El escepticismo y su refutación no son un simple modelo de cómo debe conducirse la filosofía; son más importantes que un modelo o analogía [...] La filosofía no tiene la última palabra, pero solo porque el escepticismo siempre retorna" (p. 311).

Finalmente, Morgan (2007) plantea una salida original a la aparente inviabilidad racional que la 'analogía' parece contener:

La dualidad a la que el escepticismo -y la filosofía y el lenguaje- llama la atención está entre el acto de retar la pretensión de conocimiento del creyente, de todo creyente, y la responsabilidad por el creyente que está implicada en la respuesta al llamado o mandato del creyente (p. 312).

En últimas, el escéptico, al plantear sus dudas al creyente, "siempre está respondiéndole" (lo que es una forma de ser responsable de él).

En línea con la segunda tendencia interpretativa, también se podrían situar los trabajos de García-Baró (2007) y Rosato (2015); ambos plantean una tesis similar, aunque de maneras expositivas muy distintas. La analogía de la alteridad con el escepticismo estaría respondiendo no tanto a Derrida sino al Prefacio a Totalidad e Infinito (1961), en especial, a la patencia de la guerra: "¿No es el punto de vista escéptico el contrapeso de la experiencia de la realidad como guerra, más bien que su necesario complemento?" (García-Baró, 2007, p. 111); "En Escepticismo y razón, Levinas muestra cómo la explicación del sentido y el lenguaje que ha desarrollado en De otro modo que ser responde a la sugerencia del escéptico moral que el sentido es imposible aparte de la totalidad" (Rosato, 2015, p.438).

Ahora bien, como ejemplo de la tercera tendencia interpretativa, consistente en propuestas novedosas que permiten lecturas de la obra de Levinas más allá de la exégesis, puede mencionarse el trabajo de Aikin y Simmons
(2009). A diferencia de algunos de los analíticos de Levinas, quienes sabían tanto o menos que Levinas acerca del escepticismo, Scott F. Aikin y J. Aaron Simmons se destacan por ser conocedores tanto del escepticismo como de la filosofía levinasiana.

Dado el espacio otorgado para el presente artículo, no es posible exponer en detalle la línea argumentativa de estos autores, más sí mencionar sus tres tesis: 1) "Si Levinas concede que su visión y la del escéptico están amenazadas por auto derrota, los escépticos no tienen que conceder esto"; 2) "La defensa de Sexto [Empírico] contra la autorrefutación, aunque funciona para los escépticos pirrónicos, no funciona para el otrismo de Levinas"; finalmente, 3) "Aunque la explicación levinasiana no captura una concepción escéptica más robusta (y más susceptible de defensa), la explicación revela una característica de la teoría levinasiana del significado que podría resolver la autorrefutación" (Aikin y Simmons, 2009, p. 31). Con respecto a la tercera tesis, se ha de destacar la conclusión a la que llegan sus análisis técnicos. Después de establecer algunas distinciones entre autorrefutación operacional y autorrefutación performativa (a la que pertenecería la propuesta de Levinas) y, a su vez, distinguiendo entre "referencia de re" y "referencia de dicto", los autores plantean que la autorrefutación funciona cuando se trata de operadores transparentes, como "conocer" o "probar", pero no lo hacen en el nivel de los operadores no transparentes, como "desear", "creer" o "esperar". "El mejor operador para la filosofía levinasiana es confiar (trusting)" (Aikin \& Simmons, 2009, p. 48). Este operador le permitiría al "otrismo levinasiano" una justificación ética fundamentalista (en sentido débil), entendida como la base no prescindible del pensamiento ético genuino, lo que no implica un blindaje a la crítica; todo esto lo remarcan las nociones de "testimonio, profecía y plegaria, que aparecen en toda la obra madura de Levinas" (p. 49). Retomando la discusión entre decir y dicho, mencionada a lo largo de este artículo, y sumándole lo alcanzado en el análisis 
de la confianza como operador no transparente, los autores concluyen:

La relación con el otro debe ser de confianza e investidura precisamente porque la expresión nunca se adecua al encuentro. La confianza es el mejor modo que tenemos de "expresar" esta inadecuación porque mantiene abierta la posibilidad de estar equivocado, a la vez que realiza perpetuamente el requisito del compromiso. Somos falibles y la confianza es un riesgo. Así pues, la garantía para una pretensión de confianza que uno tenga a menudo es inadecuada u opaca en cierto grado -“excede" la comprensión, no es algo sobre lo que uno razone de un solo modo (completamente) sino hacia lo que uno se lanza (pero no necesariamente sin ninguna razón) (p. 52).

El recorrido por estas tres tendencias ha permitido considerar la analogía entre alteridad y escepticismo, oteando sus distintos horizontes interpretativos. Si bien no hay una versión canónica de dicha analogía (al contrario, parece haber varios desacuerdos), cada uno de los teóricos señala nuevas rutas, lo que indica la prolijidad del texto, así como la dificultad para desentrañar su sentido y, ante todo, la necesidad de introducir elementos epistemológicos en la discusión sobre la propuesta ética levinasiana.

\section{Conclusión}

La consideración de la analogía entre la alteridad y escepticismo, hecha por Levinas en la sección "Escepticismo y razón", permite trascender la cuestión acerca de si es una respuesta o no al reto planteado por Derrida en 1964, aunque hay razones para sospechar que Levinas tuvo presente la contundente crítica de su amigo filósofo. Por otra parte, la multitud de interpretaciones que tal sección ha propiciado permite comprender que se trata de una pieza filosófica necesitada de estudio constante. Es posible que la idea que Levinas tenía de escepticismo le llevara a comparar de manera ligera su propuesta con una versión simplista de esa corriente filosófica, siendo sospechoso que con- cediera el carácter de autorrefutación al escepticismo y, de igual manera, aplicando este carácter a su propuesta de la alteridad. En cualquier caso, al margen de esta sólida acusación, la analogía suscita una serie de posibilidades que rompen los esquemas con los que se ha abordado tanto al otro como a la razón en Occidente.

Del presente estudio se coligen las siguientes perspectivas:

I. En primer lugar, la analogía introduce el reto/riesgo de poner constantemente en cuestión los discursos que se condensan como verdades. Así como el escepticismo siempre retorna en la historia de la filosofía y la figura del escéptico incomoda a quien confía en la posibilidad del conocimiento, quien investiga sobre lo humano debe asumir el vértigo de convivir con la incertidumbre. La duda respecto a si lo que afirma en su discurso se corresponde de algún modo con el "otro" que trata de tematizar, antes que llevarlo a renunciar al discurso, exige la constante reformulación de su decir y el esfuerzo por ser cada vez más preciso, así sepa que la tarea infinita nunca se colma. La exigencia y el esfuerzo se corresponden con la responsabilidad por "el otro", previa a todo ejercicio especulativo. En cuanto a la racionalidad, esta perspectiva despierta del sueño dogmático de creer que es posible la formulación de una verdad definitiva, e instaura una actitud de permanente vigilancia ante aquello que se entroniza como verdadero; dicho de otra manera, devuelve ${ }^{11}$ a la razón su carácter inquieto, de movimiento, de deseo infinito de conocer.

II. La analogía promueve la posibilidad de ubicar las fuentes del conocimiento más allá (o más acá) de lo que se toma oficialmente como evidencia. Tal y como se infiere del trabajo de Aikin y Simmons, por lo menos en la indagación sobre lo humano, las relaciones con "el otro" no se reducen a hechos constatables (modo común de abordar la evidencia); antes de esto, se da el encuentro, provisto de confianza, que, contrario a la "certeza clara y manifiesta de la que no se 
puede dudar"12, es una garantía "inadecuada" y "opaca", esto es, abierta a una duda imposible de eludir. En tal sentido, pese a la pretensión moderna por las "ideas claras y distintas", la analogía levinasiana recuerda que una razón auténtica tendrá que admitir que hay aspectos enigmáticos que siempre la desafiarán a buscar nuevos modos de abordarlos o enunciarlos.

III. La crítica levinasiana a los enunciados que pretenden agotar el sentido de lo externo al sujeto, condensada en su conceptualización de lo dicho, proclama, en la analogía con el escepticismo, la necesidad de alterar los esquemas en los que el sentido se agota, siendo sensibles a nuevos modos de expresión. En las Ciencias humanas, por ejemplo, estará siempre la necesidad de redecir lo humano ${ }^{13}$. Si el término $\mathrm{ra}$ zón se define como facultad de discurrir (entre otras acepciones), y si uno de los sentidos de discurrir es inventar o idear algo, es necesario que a la racionalidad se le saque de los petrificados moldes en los que se le trata de mantener, y explorar creativamente otras maneras de decir que hagan justicia a la experiencia de la exterioridad. Este es quizá el mayor reto que formula el escéptico: volver a decirle, responderle de nuevas maneras, esforzarse por que entienda, encontrar el sentido en sus formulaciones aparentemente sin sentido. Tal vez esto solo sea posible si, además de situarse en el ámbito de las razones, se responde "al otro", por ejemplo el escéptico, bajo la única razón de ser absolutamente otro, esto es, en radical alteridad.

\section{Notas}

1 Bernasconi (2005) recuerda que, "en una nota a "Dios y la filosofía", Levinas explicó que su ensayo era una pregunta no por la ética, sino por el significado de la trascendencia" ( $p$. 101). Según Bernasconi, a Levinas le ocurrió lo mismo que a Colón, quien puso un pie en un continente diferente al que estaba buscando; no obstante, a diferencia de Colón, "cuando [Levinas] encontró la relación ética, sabía que había llegado" (p. 101).
${ }^{2}$ En un texto relativamente reciente, Atterton y Calarco (2010) plantean que actualmente se da una 'tercera ola' en la recepción de Levinas. Las dos primeras estuvieron marcadas por la exégesis a sus dos obras principales (Totalidad e infinito, de 1961, y De otro modo que ser o más allá de la esencia, de 1974); la tercera estará cada vez más marcada por cuestiones sociales y políticas. Es, cuando menos, sorprendente este descubrimiento en el contexto anglosajón, toda vez que esta recepción social y política de Levinas se hizo hace varias décadas entre los filósofos latinoamericanos. Una contundente crítica a este respecto se encuentra en Maldonado (2012).

3 Véase la entrada "Scepticisme" en Ciocan y Hansel (2005).

${ }^{4}$ Tal y como hoy se sabe, algunos de los manuscritos de Husserl abordan ampliamente estos asuntos; no obstante, Levinas se propuso considerar en su trabajo solo la obra publicada del filósofo (que en ese momento era realmente escasa). Por consiguiente, los actuales estudiosos de Husserl podrían controvertir esta afirmación de Levinas (aunque esto no implica, necesariamente, que la crítica se desvanezca).

${ }^{5}$ Se ha de señalar, sin embargo, que en el artículo "Énigme et phénomène" (1965/1967) hay una mención que podría tomarse como preconizadora de lo posteriormente desplegado en $A E$. Allí se lee: "Nudo de una intriga que se distancia de la aventura del ser buscada en el fenómeno y en la inmanencia, modalidad nueva que se dice mediante ese "si así se quiere" y ese "quizá" y que no cabe remitir a la posibilidad, a la realidad y a la necesidad de la lógica formal a las que se refiere el escepticismo" (p. 209). En este punto, podría comenzar a tejerse un tipo distinto de relación entre la "modalidad nueva" hacia la que apunta Levinas y aquella modalidad (se podría decir antigua, esto es, la tradición), que estaría sintetizada por la "posibilidad", la "realidad" y la "lógica formal", capaz de tachar de "absurdo" al escepticismo. Podría 
sospecharse, con base en este texto de 1965, que la "modalidad nueva" tendría que adoptar una postura distinta frente al escepticismo que pretende refutado la modalidad antigua.

${ }^{6}$ Términos tomados de la segunda acepción que sobre "refractario, ria", indica el Diccionario de la lengua española - Real Academia Española (DRAE), en su versión en línea: "Opuesto, rebelde a aceptar una idea, opinión o costumbre".

7 En su uso histórico, la agogé se refería a la educación militar obligatoria para los espartanos; dicha educación la recibían cuando sus edades oscilaban entre los 7 y los 18 años. No obstante, Lamarche (2008) utiliza este término en el contexto en que lo usó el escéptico Sexto Empírico, a saber, como "un estilo de vida caracterizado por la libertad de creencia, lo que trae consigo la liberación de la perturbación" (p. 169).

${ }^{8}$ Un caso exitoso a este respecto lo constituye el texto de Soren Overgaard (2007), Rethinking Other Minds: Wittgenstein and Levinas on Expression, donde se expone una forma de evadir el reto que el escéptico plantea a la posibilidad del conocimiento de las otras mentes. Overgaard concluye que tanto Wittgenstein como Levinas ofrecen alternativas a las clásicas respuestas que se han dado a este problema epistemológico.

${ }^{9}$ No obstante, Hutchens (2004) es realista al respecto: primero afirma: "Simplemente no nos ha dado [Levinas] razones para creer que el privilegio de los temas sobre las expresiones debería reversarse". Y líneas abajo concluye: "Es altamente improbable que la epistemología adopte la hipérbole decir/dicho o integre su investigación sobre la naturaleza del escepticismo con el juego dialógico" (p. 66). Más que desanimar a los epistemólogos, estas afirmaciones de Hutchens los exhorta a "dar las razones" que fueron esquivas a Levinas, así como para ver de qué manera sus planteamientos tendrían cabi- da en discusiones contemporáneas. El caso de Aikin y Simmons $(2009,2012)$ es una muestra de ello.

10 Agradezco al Doctor Germán Vargas Guillén haberme planteado un comentario en esta misma dirección, lo que me permitió comprender de modo más preciso y desde una perspectiva metodológica, algunos textos levinasianos.

${ }^{11}$ No es extraño que una de las acepciones de "Renovar" sea, precisamente, hacer como de nuevo algo, o volverlo a su primer estado. De esto se puede inferir que, si en el origen del razonamiento estaba el asombro, la recurrencia de Levinas al escéptico promueve la concepción de una racionalidad que no queda estupefacta frente a una verdad dicha, esto es, sugiere una racionalidad que aún en lo dicho se sigue maravillando por las nuevas preguntas que lo dicho inaugura.

${ }^{12}$ Definición del DRAE para "Evidencia”.

13 Aguirre (2017) plantea un intento por desplegar esta idea a partir de las tesis de Levinas.

\section{Referencias}

Aguirre, J. (2017). Redecir lo humano. Revista Colombiana de Educación 72, 177-197. doi: 10.17227/01203916.72rce177.196

Aikin, S., \& Simmons, A. (2009). Levinasian Otherism, Skepticism, and the Problem of Self-refutation. The Philosophical Forum 40(1), 29-54. doi: 10.1111/j.14679191.2008.00315.x

Aikin, S., \& Simmons, A. (2012). Prospects for a Levinasian Epistemic Infinitism. International Journal of Philosophical Studies 20(3), 437-460. doi: 10.1080/09672559.2012.697285

Atterton, P., \& Calarco, M. (2010). Editors' introduction. The third way of Levinas 
Scholarship. En P. Atterton \& M. Calarco (Eds.), Radicalizing Levinas (pp. 4-17). Albany: Suny Press.

Bernasconi, R. (1991). "Skepticism in the Face of Philosophy". En R. Bernasconi \& S. Critchley (Eds.), Re-reading Levinas (pp. 149-161). Bloomington: Indiana University Press.

Bernasconi, R. (2005). No Exit: Levinas' aporetic account of transcendence. Research in Phenomenology, 35(1), 101117. doi: 10.1163/1569164054905366

Ciocan, C., \& Hansel, G. (2005). Levinas Concordance. Dordrecht: Springer.

Critchley, S. (2004). Introduction. En S. Critchley \& R. Bernasconi (Eds.), The Cambridge Companion to Levinas (pp. 1-32). Cambridge: Cambridge University Press.

Derrida,J.(Ed.).(1967).Violence etMétaphysique. Essai sur la pensé d'Emmanuel Levinas. En, L'écriture et la différence (pp. 117228). Paris: Éditions du Seuil.

García-Baró, M. (2007). El desafío de Levinas. Anuario Colombiano de Fenomenología, 1, 107-119. Recuperado de http://www. filosofiayensenanza.org/inicio/images/ CONTENIDO/ANUARIO/Anuario $\% 20$ Colombiano\%20de\%20fenomenologa $\% 20$ Vol\%20I.pdf

Hutchens, B. (2004). Levinas: A Guide For the Perplexed. Nueva York: Continuum.

Klein, P. (2015). Skepticism. En E. Zalta (Ed.), The Stanford Encyclopedia of Philosophy.
Recuperado de http://plato.stanford.edu/ archives/sum2015/entries/skepticism/

Lamarche, P. (2008). Of a non-saying that says nothing: Levinas and Pyrrhonism. En B. Schroeder \& S. Benso (Eds.), Levinas and the Ancients (pp. 165-177). Bloomington: Indiana University Press.

Lemos, N. (2007). An introduction to the Theory of Knowledge. Cambridge: Cambridge University Press.

Levinas, E. (1967). En Découvrant l'existence avec Husserl et Heidegger (2 ed.). Paris: Vrin.

Levinas, E. (1974). Autrement qu'être ou au-delà de l'essence. La Haya: Martinus Nijhoff.

Levinas, E. (2001). Théorie de l'intuition dans la phénoménologie de Husserl. París: Vrin.

Maldonado, N. (2012). Levinas's hegemonic identity politics, radical philosophy, and the unfinished project of decolonization. Levinas Studies 7, 63-94. doi: 10.5840/ levinas 201276

Morgan, M. (2007). Discovering Levinas. Cambridge: Cambridge University Press.

Overgaard, S. (2007). Rethinking Other Minds: Wittgenstein and Levinas on Expression. Nueva York: Routledge.

Real Academia Española (2014). Diccionario de la lengua española (23a ed.). Recuperado de http://dle.rae.es/?id=W0DqCvJ

Rosato, J. (2015). Levinas on Skepticism, Moral and Otherwise. Philosophy Today 59(3), 429-450. doi: 10.5840/philtoday201561076 